\title{
Humoralpathologische Diagnostik in der Konstitutionellen Altorientalischen Musiktherapie
}

\author{
Michael Bachmaier-Ekşi \\ Praxis für Orientalische Medizin, DE-Berlin
}

D er Begriff der „altorientalischen Musiktherapie" wurde in den 80er Jahren von einer Arbeitsgruppe um Dr. Oruç Güvenç, dem Leiter des Zentralasieninstituts der Marmara Universität Istanbul, und dabei insbesondere von dem Wiener Musiktherapeuten GERNot G. STANFEL, in die musiktherapeutische Landschaft Europas eingeführt und bezeichnet eine Gattung der Musiktherapie, deren Wurzeln in der Kultur und Tradition des Orients zu finden sind. Der Begriff umfasst die klassisch-arabische und später osmanische Musiktherapie, die aufgrund ihrer konstitutionsmedizinischen Ausrichtung vom Autor als „konstitutionelle altorientalische Musiktherapie" bezeichnet wird, die zentralasiatische Musiktherapie mit ihren verschiedenen Bewegungs- und Tanztherapieformen sowie Therapievarianten, die von einzelnen Therapeuten in den letzten Jahren aufgrund eigener Erfahrungen entwickelt worden sind. Seit den 80er Jahren wird die altorientalische Musiktherapie als mittlerweile professionalisierte Therapie in Europa gelehrt und überwiegend von Ärzten, Psychologen und Musiktherapeuten in Zusammenarbeit mit speziell ausgebildeten Musikern europaweit therapeutisch eingesetzt [1-3].

Die altorientalische Musiktherapie war bis ins 18. Jahrhundert hinein integrierte Behandlungsmethode an zahlreichen medizinischen Zentren (türk.: şifahane) der islamischen Welt, die mit der Idee einer empathischen, standesunabhängigen Sozialfürsorge ab dem 8. Jahrhundert ein niedrigschwelliges, gut strukturiertes öffentliches Gesundheitswesen etablierte. Sie

In der Konstitutionellen altorientalischen Musiktherapie werden verschiedene Temperamente mit verschiedenen Tonalitäten (Makamen) behandelt. Methodische Grundlage ist nach den Thesen des Autors die Humoralmedizin, wie sie vor 1000 Jahren von Ibn Sina praktiziert wurde und heute als moderne Regulationsmedizin einen funktionellen Ansatz vertritt. Um die Altorientalische Musiktherapie in ihrer historisch überlieferten Form anwenden zu können, hat der Autor einen Befunderhebungsbogen entwickelt, der sowohl traditionelle als auch moderne Aspekte der Konstitutionsmedizin berücksichtigt. Dabei zeigt sich eine weitgehende Übereinstimmung zwischen der Humoralmedizin und der Traditionellen Chinesischen Medizin (TCM).

Schlüsselwörter: Konstitutionelle altorientalische Musiktherapie, Temperament, Makam, Humoralmedizin, Ibn Sina, Regulationsmedizin, Traditionelle Chinesische Medizin (TCM)

\section{Humoural Diagnosis in Constitutional Oriental Music Therapy}

In Constitutional Oriental Music Therapy, different temperaments are treated by different tonalities (makams). According to the author's thesis, its methodical basis is humoural medicine as practiced by Ibn Sina 1000 years ago. Today, humoural medicine appears as a modern form of regulatory medicine. To employ Oriental Music Therapy in an original way, the author developed a detailed method of anamnesis which considers traditional and modern aspects of 'constitutional' medicine. There is a far-reaching conformity between humoural medicine and Traditional Chinese Medicine.

Keywords: Constitutional Oriental Music Therapy, temperament, makam, humoural medicine, Ibn Sina, regulatory medicine, Traditional Chinese Medicine

ist das wissenschaftliche Erbe arabischer Ärzte und Musiktheoretiker, wie Ibn Sina (Abb. 1), Al Kindi und $\mathrm{Al}$ Farabi. Viele Krankenhäuser beschäftigten bereits vor 1000 Jahren eigene Orchester, die die ärztliche Verordnung von Musiktherapie ausführten, um die Patienten mit nach humoraldiagnostischen Prinzipien ausgewählten Musikrezepten zu behandeln. Historische Texte weisen auf die Verknüpfung von ärztlicher Diagnostik und Musiktherapie hin: „,Es wird berichtet, dass im arabischen Spanien (alAndalus) Ärzte, die musikalisch gebildet sind (arifun bi-l-musiqa) den Puls des Kranken fühlen und in einem Modus (maqam), der ihm entspricht, für den Kranken singen. Dadurch nehmen sie ihm den grössten Teil seiner Schmerzen” “ [4] (Abb. 2/3).

Ziel dieser Arbeit ist es, die medizinischen Hintergründe der konstitutionellen altorientalischen Musiktherapie anhand eigener Thesen zu vermitteln. Dabei soll auf die Gemeinsamkeiten der europäischen, arabischen und chinesischen Medizintradition hingewiesen werden. Dem interessierten Musiktherapeuten gibt der Autor hier erstmalig ein eigens entwickeltes praktikables Diagnosekonzept an die 


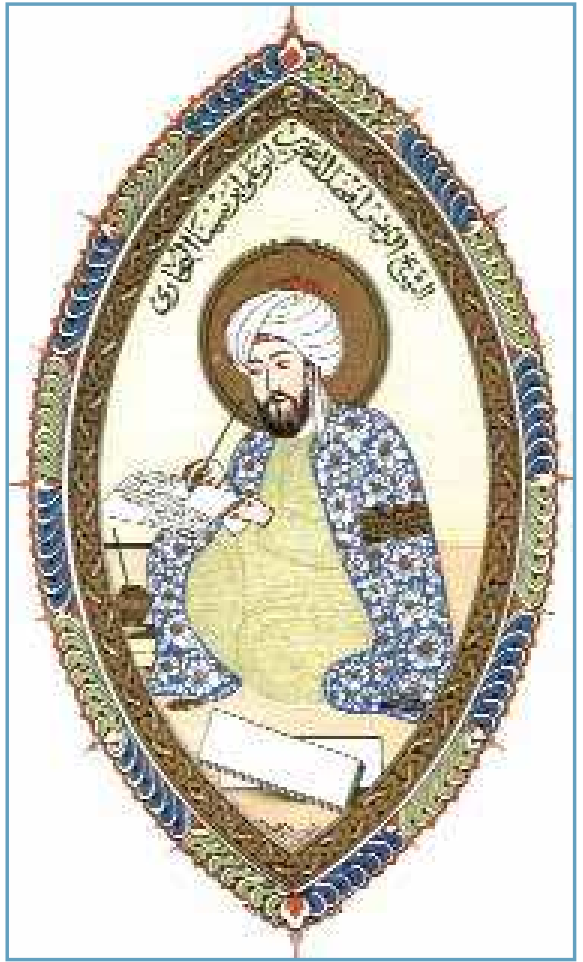

Abb. 1. Miniatur von lbn Sina (980-1037 n. Chr.), genannt Avicenna, Arzt, Philosoph und Musikwissenschaftler.

Hand, das es ihm ermöglicht, die Auswahl der für die Behandlung erforderlichen Tonalitäten auf der Grundlage der Ibn Sina'schen Medizin zu treffen.

\section{Theoretische Grundlagen}

, ,Wisse, dass die Modi (angam) aus [den] vier „Naturen“ (taba'i) zusammengesetzt (murakkeb) sind, aus Wärme (harara), Kälte (buruda), Trockenheit (yabusa) und Feuchtigkeit (rubuta) und aus deren Verbindungen gelbe Galle (safra), schwarzer Galle (sauda), Blut (dam) und Schleim (balgam) sowie aus Nacht (laila) und Tag (nahar). Der Musiker (mutrib) muss dieses wissen, damit er [jeweils] in dem Modus singt, der [der Situation] entspringt.' " [4]

Durch diese syrische Quelle aus dem 14.-16. Jahrhundert (Anonymus Gotha) erfährt der Musiktherapeut, dass Musiktherapie ohne medizinisches Basiswissen nicht lege artis ausgeführt werden kann. In einem unpassenden Makam zu musizieren, galt zu damaliger Zeit nicht weniger schädlich als die Verabreichung eines falschen Medikaments. Denn wie einem Medikament wurde auch einem bestimmten Klang eine direkte und differenzierte Wirkung auf den Körper und die Psyche des Menschen zugeschrieben. Der diagnostische Prozess musste dementsprechend mit grosser Sorgfalt und auf der Grundlage des damals vorherrschenden Medizinsystems betrieben werden.

Mit dem 9. Jahrhundert entwickelte sich die klassisch-griechische Medizintheorie im arabischen Raum zur Grundlage der wissenschaftlichen Medizin. Im „Kanon der Medizin“ (Al Qanon Fit-Tibb) bezieht sich Ibn Sina (980-1037 n. Chr.) auf den römischen Arzt Galen (131-210 n. Chr.), der vier Körpersäfte (lat.: humores) differenzierte, die Konstitution und Gesundheit eines Menschen bestimmten: Blut (Sanguis), Gelbgalle (Cholera), Schleim (Phlegma) und Schwarzgalle (Melancholera). Die Dominanz eines dieser Säfte sei bestimmend für sein charak-

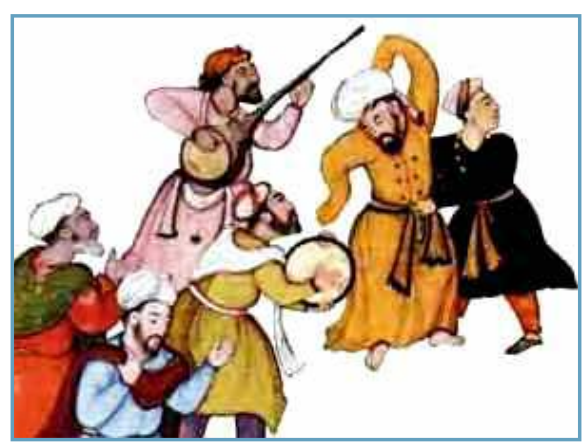

Abb. 2. "Die mystische Bruderschaft" -

Musiker begleiten einen Derwisch-Tanz. Detail einer Mughul-Miniatur aus dem 16. Jahrhundert.

teristisches „Temperament“. Befanden sich die Säfte in einem harmonischen Gleichgewicht (arabisch: mizaj-emutadil), erschien der Mensch gesund. Kam es zum Ungleichgewicht der Säfte (arab.: mizaj-e-ghair mutadil), erschien die Konstitution geschwächt und der Mensch wurde krank.

Die Lehre von den Körpersäften (Humoralpathologie) war eng verbunden mit der Lehre von den Vier Elementen (arab.: arkan), die seit dem 4. Jahrhundert v. Chr. den Natur- und Geisteswissenschaften als gemeinsame

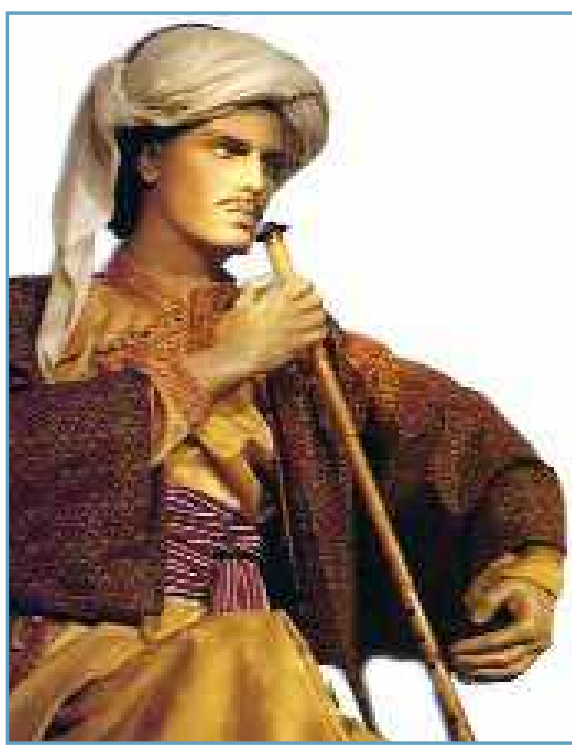

Abb. 3. Musiktherapeut mit Rohrflöte (Ney) Installation des Medizinischen Museums Edirne.

Grundlage diente. Jedes fassbare Phänomen der Natur liess sich auf vier Grundbausteine reduzieren. Diese so genannten Elemente waren Luft, Feuer, Wasser und Erde. Sie wurden durch vier Sinnesqualitäten charakterisiert: warm, kalt, feucht und trocken. Luft galt als „warm und feucht“, Feuer hingegen als „warm und trocken“. Wasser galt als „kalt und feucht“, Erde als „kalt und trocken“. Die Beziehung zwischen Elementen und Temperamenten zeigt Tabelle 1 (arabische Bezeichnungen in Klammern).

Mit der Lehre von den vier Elementen konnten verschiedenste Faktoren des Mikro- und Makrokosmos in das diagnostische und therapeutische Denken integriert werden. Dies führte zur einer Medizinpraxis, die bei der Behandlung biologische, psychologische und soziale Faktoren gleichermassen berücksichtigte. (Tabelle 2).

\section{Moderne Humoralmedizin}

Nach den Vorstellungen des Autors lässt sich Humoralmedizin heute als Regulationsmedizin verstehen, Humoralpathologie als „biokybernetisches Arbeitsmodell“ [8]. Die so genannte Konstitutionsmedizin versucht mit überwiegend ganzkörperlich wirkenden Methoden, wie Diätetik, Schröp- 
Tab. 1. Beziehung zwischen den Elementen und Temperamenten $[5,6,11]$

\begin{tabular}{|l|l|l|l|l|}
\hline Elemente (arkan) & Luft (hawa) & Feuer (nar) & Wasser (ma) & Erde (arz) \\
\hline $\begin{array}{l}\text { Qualitäten } \\
\text { Körpersäfte } \\
\text { (akhat) }\end{array}$ & $\begin{array}{l}\text { Blut } \\
\text { (har wa ratab) }\end{array}$ & $\begin{array}{l}\text { warm-trocken } \\
\text { (har wa yabis) }\end{array}$ & $\begin{array}{l}\text { kalt-feucht } \\
\text { (barid wa ratab) }\end{array}$ & $\begin{array}{l}\text { kalt-trocken } \\
\text { (bard wa yabis) }\end{array}$ \\
\hline $\begin{array}{l}\text { Temperamente } \\
\text { (mizaj) }\end{array}$ & $\begin{array}{l}\text { Sanguiniker } \\
\text { (damwi mizaj) }\end{array}$ & $\begin{array}{l}\text { Choleriker } \\
\text { (safrawi mizaj) }\end{array}$ & $\begin{array}{l}\text { Shlegmatiker } \\
\text { (balghami mizaj) }\end{array}$ & $\begin{array}{l}\text { Melancholiker } \\
\text { (baudawi mizaj) }\end{array}$ \\
\hline
\end{tabular}

Tab. 2. Medizinisch relevante Aspekte der Vier-Säfte-Lehre $[5,6,7,11]$

\begin{tabular}{|c|c|c|c|c|}
\hline Element & Luft & Feuer & Wasser & Erde \\
\hline $\begin{array}{l}\text { Elementare } \\
\text { Fähigkeiten }\end{array}$ & $\begin{array}{l}\text { Erweiterung, } \\
\text { Verdünnung, } \\
\text { Erleichterung }\end{array}$ & $\begin{array}{l}\text { Reifung, } \\
\text { Durchdringung, } \\
\text { Verfeinerung }\end{array}$ & $\begin{array}{l}\text { Umwandlung, } \\
\text { Anpassung, } \\
\text { Flexibilisierung }\end{array}$ & $\begin{array}{l}\text { Strukturgebung, } \\
\text { Formwahrung, } \\
\text { Stabilisierung }\end{array}$ \\
\hline Qualität & warm-feucht & warm-trocken & kalt-feucht & kalt-trocken \\
\hline Temperament & sanguin & cholerisch & phlegmatisch & melancholisch \\
\hline Kardinalsaft & Blut & Gelbgalle & Schleim & Schwarzgalle \\
\hline Hauptorgan & Herz & Leber & Gehirn & Milz \\
\hline Gewebe & Gefässe & $\begin{array}{l}\text { Muskeln } \\
\text { Sehnen }\end{array}$ & $\begin{array}{l}\text { Fett } \\
\text { Bindegewebe }\end{array}$ & Knochen \\
\hline Körperfunktion & Atmung & Verdauung & Ernährung & Struktur \\
\hline Sinnesorgane & Gehör & Geruch & Geschmack & Berührung \\
\hline Grundstimmung & $\begin{array}{l}\text { Ausgelassen- } \\
\text { heit }\end{array}$ & $\begin{array}{l}\text { Entschlossen- } \\
\text { heit }\end{array}$ & Gelassenheit & $\begin{array}{l}\text { Nachdenklich- } \\
\text { keit }\end{array}$ \\
\hline Emotion & Freude & Wut & Innere Ruhe & Angst \\
\hline $\begin{array}{l}\text { Traummotiv } \\
\text { (z.B.) }\end{array}$ & $\begin{array}{l}\text { Schwere Las- } \\
\text { ten, Blut }\end{array}$ & $\begin{array}{l}\text { Krieg, } \\
\text { Flammen }\end{array}$ & $\begin{array}{l}\text { Reinigung, } \\
\text { Wasser, Eis }\end{array}$ & $\begin{array}{l}\text { Dunkelheit, } \\
\text { Schrecken }\end{array}$ \\
\hline Lebensalter & Kindheit & Jugend & Alter & Erwachsensein \\
\hline Nahrung & süss & bitter / scharf & salzig & sauer \\
\hline Farbe & rot & gelb & weiß & schwarz \\
\hline Tageszeit & Morgen & Mittag & Nacht & Abend \\
\hline Jahreszeit & Frühling & Sommer & Winter & Herbst \\
\hline Himmelskörper & Merkur & Mars, Sonne & Jupiter, Mond & Venus, Saturn \\
\hline
\end{tabular}

Tab. 3. Humoralmedizinische Normkonventionen $[1,6]$

\begin{tabular}{|l|l|l|}
\hline \multirow{2}{*}{ viel } & Aktivität (Energie) & Struktivität (Körperlichkeit) \\
\hline wenig & $\begin{array}{l}\text { "warm” } \\
\text { (z.B. schnell, aktiv, rot, warm) }\end{array}$ & $\begin{array}{l}\text { "feucht” } \\
\text { (z.B. voll, reichlich, kräftig, feucht) }\end{array}$ \\
\hline $\begin{array}{l}\text { "kalt” } \\
\text { (z.B. langsam, passiv, weiss, kalt) }\end{array}$ & $\begin{array}{l}\text { "trocken“ } \\
\text { (z.B. leer, spärlich, schwach, trocken) }\end{array}$ \\
\hline
\end{tabular}

fen, Akupunktur, Aderlass oder Eigenbluttherapie, die Regulationsvorgänge im Körper so zu beeinflussen, dass Krankheiten verhindert oder leichter überwunden werden können. Das Modell der Körpersäfte dient dabei, wie auch das Konzept des chinesischen $Q i$, der Darstellung komplexer Regelmechanismen im menschlichen Körper. Gesundheit beschreibt einen Zustand von Ausgeglichenheit, in dem der Mensch auf Einflüsse seiner Um- welt adäquat reagiert. Für diesen Zustand verwendet die arabische Humoralmedizin den Begriff des „ausgewogenen“ oder „natürlichen Temperaments “ (arab.: mizaj-e-mutadil). Das natürliche Temperament beschreibt ein qualitativ bestimmbares gesamtkörperliches Funktions- und Reaktionsniveau. Dieses gibt Aufschluss über die Reaktionskapazitäten sowie die zu erwartende Fehlregulation. Die vier Körpersäfte (arab.: akhat) lassen sich als „Wirkprinzipien“ interpretieren, deren divergierende Kräfte das physiologische System in einem dynamischen Gleichgewicht stabilisieren.

Traditionelle Begriffe, wie "Yin“ und „Yang“, „Fülle“ und „Leere“, „Hitze“ und „Kälte“ beschreiben das polare Verhältnis der energetischen und materiellen Komponente eines biologischen Systems, also der Beziehung von „Aktivität“ und „Struktivität“ [9]. Ausgehend von einem hypothetischen Idealzustand bezeichnen die Normkonventionen „warm“ und „kalt“ (in energetischer Hinsicht) und „feucht“ und „trocken“ (in materieller Hinsicht) die Wirkrichtung, in welche Einflussfaktoren das physiologische System von seinem angenommenen Ruhepunkt ablenken (Tabelle 3).

Aus den vier möglichen Kombinationen der Qualitäten „warm-feucht“, „warm-trocken“, „kalt-feucht“ und „kalt-trocken“ ergeben sich die vier Temperamente (Tabelle 4).

„Krankheit“ wird im traditionellen Sinne als eine Abweichung von der gesunden Mitte definiert. Sie wird diagnostiziert, wenn das Temperament durch die pathologische Dominanz eines der vier Kardinalsäfte ins Ungleichgewicht geraten ist (arab.: mizaj-e-ghair mutadil). Zur modernen Beschreibung der klassischen Dysregulationsmuster schlägt der Autor eine Klassifizierung vor, wie sie in Tabelle 5 dargestellt ist.

\section{Humoralpathologische Diagnostik}

Jede der genannten Normkonventionen entspricht einer Gruppe von Symptomen und Einflussfaktoren, aus denen sich das Temperament zusam- 
Tab. 4. Temperamente als Ausdruck von Aktivität und Struktivität [1]

\begin{tabular}{|l|l|}
\hline $\begin{array}{l}\text { Überschuss an Aktivität (warm) } \\
\text { Überschuss an Substrat (feucht) } \\
=\text { sanguines Temperament }\end{array}$ & $\begin{array}{l}\text { Überschuss an Aktivität (warm) } \\
\text { Mangel an Substrat (trocken) } \\
\text { = cholerisches Temperament }\end{array}$ \\
\hline $\begin{array}{l}\text { Mangel an Aktivität (kalt) } \\
\text { Überschuss an Substrat (feucht) } \\
=\text { phlegmatisches Temperament }\end{array}$ & $\begin{array}{l}\text { Mangel an Aktivität (kalt) } \\
\text { Mangel an Substrat (trocken) } \\
=\text { melancholisches Temperament }\end{array}$ \\
\hline
\end{tabular}

Tab. 5. Muster der Dysregulation

\begin{tabular}{|l|l|l|l|}
\hline Sanguine Entgleisung & $\begin{array}{l}\text { Cholerische } \\
\text { Entgleisung }\end{array}$ & $\begin{array}{l}\text { Phlegmatische } \\
\text { Entgleisung }\end{array}$ & $\begin{array}{l}\text { Melancholische } \\
\text { Entgleisung }\end{array}$ \\
\hline $\begin{array}{l}\text { hyperaktives- } \\
\text { hyperstruktives }\end{array}$ & $\begin{array}{l}\text { hyperaktives- } \\
\text { hypostruktives } \\
\text { Syndrom }\end{array}$ & $\begin{array}{l}\text { hypoaktives- } \\
\text { hyperstruktives } \\
\text { Syndrom }\end{array}$ & $\begin{array}{l}\text { hypoaktives- } \\
\text { hypostruktives } \\
\text { Syndrom }\end{array}$ \\
\hline
\end{tabular}

menfügt. Beim Studium der Quellentexte findet sich hinsichtlich der diagnostischen Leitkriterien eine weitgehende Übereinstimmung zwischen der Ibn Sina-Medizin (und ihrer Weiterführung in der indisch-pakistanischen Unani-Medizin) und der Traditionellen Chinesischen Medizin (TCM). Beide Schulen gehen bei ihrer Diagnostik den Weg der „induktiven Synthese“ [9], indem aus der Summe der Symptome und Einflussfaktoren die qualitative Beschreibung des aktuellen, funktionellen Gesamtzustandes entsteht. Die Schwierigkeit, die sich daraus ergibt, dass die TCM fünf, die Ibn SinaMedizin hingegen nur vier Elemente postuliert, löst sich bei genauerer Betrachtung der diagnostischen Leitlinien auf. Im Zentrum der „Acht diagnostischen Leitkriterien “ [10] der TCM (ba gang) stehen die vier Grundqualitäten „Hitze“, „Kälte“, „Fülle“ und „Leere“. In einem modernen, funktionellen Sinne interpretiert, entsprechen die beiden letzteren weitgehend den mit „Feuchtigkeit“ und „Trockenheit“ in der Ibn Sina-Medizin beschriebenen Qualitäten, nämlich dem Ausmass körperlichen Potentials und Reagibilität.

Der hier vorgestellte Befunderhebungsbogen (Auszüge in Tabelle 6) einer systemübergreifenden, ganzheitlichen Diagnostik orientiert sich weitgehend an Ibn Sina's „Kanon der

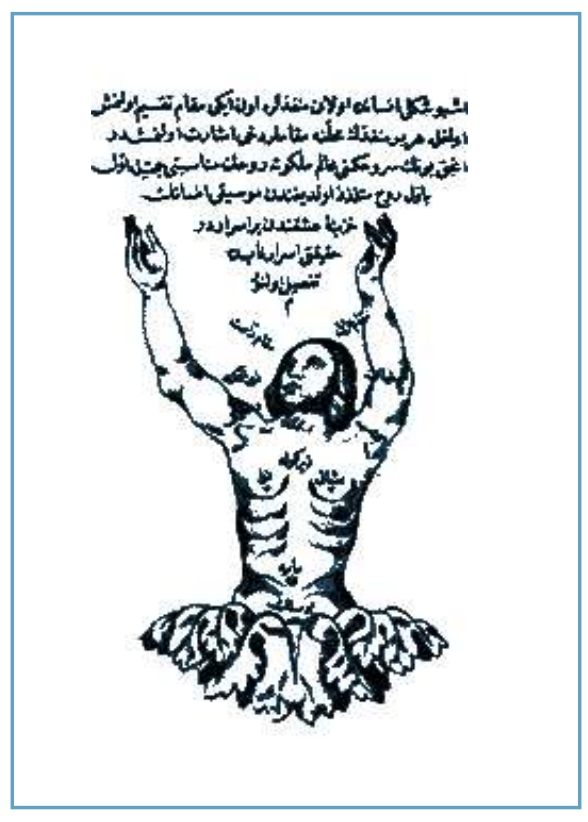

Abb. 4. Darstellung der Zuordnung der Makamen zu den verschiedenen Körperregionen (aus einem alten osmanischen Buch).

Medizin“, an diagnostischen Kriterien der Unani-Medizin, der westlichen Humoralmedizin und der TCM, soweit sie der modernen Konzeption einer funktionell ausgerichteten Temperamentenlehre entsprechen. Die Ibn Sina-Medizin legt grossen Wert auf die Abklärung der Lebensumstände des Patienten. Sie werden unter den genannten „six essential causes“ [11] (asbab-e-sittah zaruriah) zusammengefasst: Luft (hawah), Essen und Trinken (makool-o-mashrooh), körperliche Bewegung und Erholung (harat-wasukoon badania), geistige Bewegung und Erholung (harkat-wa-sukoon nafsania), Schlafgewohnheiten (naum-oyaqzah) und Zurückhaltung und Ausscheidung (ehtibas-o-istafraagh).

\section{Makamtherapie}

Die folgende Tabelle 7 nach einer syrisch-arabischen Quelle [4] beschreibt die Indikationen verschiedener Makame (siehe auch Abb. 4). Die Einteilung erfolgte in erster Linie entsprechend des Temperaments, in zweiter Linie nach Lokalisation, Symptomen und Erkrankung.

Von den über 500 bekannten Makamen sind noch zirka 50 in musiktherapeutischer Verwendung. Sie wurden von Oruç Güvenç als Therapie-CDs veröffentlicht.

\section{Diskussion}

Die Erfahrung mit traditionellen Therapieverfahren zeigt, dass eine systemimmanente Diagnostik die Therapieergebnisse verbessert. Dies gilt für die TCM ebenso, wie für die Unani-Medizin oder konstitutionell orientierte westliche Naturheilverfahren. Ihnen liegt ein von der modernen westlichen Medizin abweichendes Krankheitsverständnis zugrunde. Während die moderne Medizin auf kausalanalytischem Weg einzelne Krankheitsfaktoren aufdeckt und auf ihre Ursachen zurückschliesst, gehen traditionelle Verfahren den Weg der induktiven Synthese zur Bestimmung des funktionellen Gesamtzustandes. Krankheiten werden als dynamische Abweichung von einem individuell spezifischen Optimum angesehen. Die Behandlung besteht nicht in der Beseitigung einzelner Symptome, sondern in der Stärkung der natürlichen Konstitution. Schwächung der Konstitution wird als eigentlicher Ausgangspunkt von Erkrankungen betrachtet.

Die moderne Grundlagenforschung bestätigt die Komplexität biologischer 
Tab. 6. Befunderhebungsbogen (nach Bachmaier-Ekşi)

\begin{tabular}{|c|c|c|c|c|}
\hline Dysharmonie & $\begin{array}{l}\text { sanguine } \\
\text { Entgleisung }\end{array}$ & $\begin{array}{l}\text { cholerische } \\
\text { Entgleisung }\end{array}$ & $\begin{array}{l}\text { phlegmatische } \\
\text { Entgleisung }\end{array}$ & $\begin{array}{l}\text { melancholische } \\
\text { Entgleisung }\end{array}$ \\
\hline TCM & $\begin{array}{l}\text { Fülle-Hitze } \\
\text { Yang-Fülle }\end{array}$ & $\begin{array}{l}\text { Leere-Hitze } \\
\text { Yin-Leere }\end{array}$ & $\begin{array}{l}\text { Fülle-Kälte } \\
\text { Yin-Fülle }\end{array}$ & $\begin{array}{l}\text { Leere-Kälte } \\
\text { Yang-Leere }\end{array}$ \\
\hline Erster Eindruck & "blühend" & "gespannt" & "weich" & "schwächlich" \\
\hline Gesicht & voll, gerötet & hager, gelblich & $\begin{array}{l}\text { teigig, gedunsen, } \\
\text { weiss }\end{array}$ & $\begin{array}{l}\text { eingefallen, } \\
\text { dunkel }\end{array}$ \\
\hline Statur & fleischig-fest & sehnig-drahtig & fett-weichlich & mager-schwach \\
\hline Haut & warm-feucht & warm-trocken & kalt-feucht & kalt-trocken \\
\hline Körperempfinden & $\begin{array}{l}\text { Schwere, Druck } \\
\text { hinter den Augen }\end{array}$ & $\begin{array}{l}\text { Hitzigkeit, } \\
\text { innere Unruhe }\end{array}$ & $\begin{array}{l}\text { Kälte, } \\
\text { Lethargie }\end{array}$ & $\begin{array}{l}\text { Mattigkeit, } \\
\text { Starre }\end{array}$ \\
\hline Bewegungen & $\begin{array}{l}\text { kräftig und rasch, } \\
\text { später: schwerfällig }\end{array}$ & $\begin{array}{l}\text { hektisch, flattrig, } \\
\text { später: kraftlos }\end{array}$ & langsam, träge & langsam, schwächlich \\
\hline Schmerz & berstend & anfallsartig & dumpf, drückend & brennend \\
\hline Verbesserung durch & Kälte und Trockenheit & Kälte und Feuchtigkeit & Wärme und Trockenheit & Wärme und Feuchtigkeit \\
\hline bevorzugte Jahreszeit & Herbst & Winter & Sommer & Frühjahr \\
\hline Emotion / Psyche & $\begin{array}{l}\text { Innerer Druck, } \\
\text { Überspanntheit, } \\
\text { Manie }\end{array}$ & $\begin{array}{l}\text { Zorn, } \\
\text { Auto-Agrgression, } \\
\text { Depression }\end{array}$ & $\begin{array}{l}\text { Gleichgültigkeit, } \\
\text { Abgestumpftsein, } \\
\text { geistiges Betäubtsein }\end{array}$ & $\begin{array}{l}\text { Angst, } \\
\text { Melancholie } \\
\text { (Gefühl der Leere) }\end{array}$ \\
\hline Verdauung & $\begin{array}{l}\text { kräftig, verträgt viel } \\
\text { Nahrung }\end{array}$ & $\begin{array}{l}\text { beschleunigt, } \\
\text { evt. Durchfall und } \\
\text { Erbrechen }\end{array}$ & $\begin{array}{l}\text { träge, Nahrung bleibt } \\
\text { im Magen liegen, } \\
\text { Blähungen, Obstipation }\end{array}$ & $\begin{array}{l}\text { exzessiver oder } \\
\text { verdorbener Appetit, } \\
\text { Magenbrennen }\end{array}$ \\
\hline Puls & $\begin{array}{l}\text { schnell, } \\
\text { kraftvoll }\end{array}$ & $\begin{array}{l}\text { schnell, gespannt, } \\
\text { drahtig }\end{array}$ & langsam, voll, weich & $\begin{array}{l}\text { langsam, } \\
\text { schwach (evt. auch hart) }\end{array}$ \\
\hline Zunge & $\begin{array}{l}\text { gerötet, feucht, gross, } \\
\text { evt. dick-gelblicher } \\
\text { Belag, evt. Geschwüre }\end{array}$ & $\begin{array}{l}\text { gerötet, trocken, rissig, } \\
\text { schmal, evt. dünn-gelb- } \\
\text { licher Belag }\end{array}$ & $\begin{array}{l}\text { blass, feucht, } \\
\text { geschwollen, weisslicher } \\
\text { bis schleimiger Belag }\end{array}$ & $\begin{array}{l}\text { dunkel, trocken, } \\
\text { fehlender Belag }\end{array}$ \\
\hline
\end{tabular}

Systeme. Vor allem multifaktorielle, funktionelle und chronische Erkrankungen entziehen sich oft der kausalanalytischen Methode. PISchinger's Untersuchungen über das „Grundsystem“ [12] des Menschen bestätigen humoralpathologische Vorstellungen hinsichtlich eines ganzkörperlichen Regulationssystems. Die von ihm beobachtete bei geeigneter Stimulation auftretende Rhythmisierung von Muskelpotentialen entsprechend der musikalischen Obertonreihe lässt vermuten, dass Musik eine direkte Wirkung auf Informations- und Regulationsprozesse im menschlichen Körper entfaltet. Die von BrüGGENWERTH et al. im EEG (u.a. durch altorientalische $\mathrm{Mu}$ sik) ausgelösten Veränderungen der Hirnrindenaktivitäten [13] zeigten unterschiedliche EEG-Signale bei unterschiedlichen Tonalitäten.
Die konstitutionelle altorientalische Musiktherapie ist neben der indischen Raga-Musiktherapie eines der differenziertesten historisch überlieferten Musiktherapie-Systeme. Sie basiert auf einem medizinisch-historischen Theoriegebäude, dessen Prinzipien die Weltmedizin über mehrere tausend Jahre kulturübergreifend geprägt haben und noch heute prägen. In der konstitutionellen altorientalischen Musiktherapie kommt es zu einer aussergewöhnlich engen Verknüpfung von Medizin und Musik. Die Anwendung der Musik ist praktisch ohne die Medizin nicht möglich. Daraus ergibt sich konsequenterweise eine Therapiepraxis, die sich von der Praxis moderner europäischer Musiktherapie, die sich zunehmend psychotherapeutischer und pädagogischer Elemente bedient, eindrucksvoll unter- scheidet. Die konstitutionelle altorientalische Musiktherapie vertraut nach wie vor auf die Wirkung des Klangs als das eigentliche heilende Medium, Sie berührt dabei die körperliche, psychische und seelische Ebene der menschlichen Existenz. Sie bedient sich einer Diagnostik, die der Komplexität des zugrunde liegenden Menschenbildes und der Komplexität des verwendeten Mediums angemessen erscheint. Ein streng wissenschaftlicher Beweis für die postulierte Wirkung der verschiedenen Makame lässt sich dabei kaum erbringen. In diesem Sinne ist die konstitutionelle altorientalische Musiktherapie „Erfahrungsmedizin“. Unsere Erfahrung zeigt, dass die altorientalische Musiktherapie in der Lage ist - in Zusammenarbeit mit den konventionellen Therapieverfahren - somatische, psychosomatische und psychi- 
Tab. 7. Indikation verschiedener Makame

\begin{tabular}{|c|c|c|c|}
\hline & Makam & wirksame Region & Symptome / Erkrankung \\
\hline \multirow[t]{3}{*}{ Sanguine Entgleisung } & Irak & Kopf, Hals, Nacken & $\begin{array}{l}\text { Fieberdelirium, Raserei, Kopfleiden, } \\
\text { Phrenitis, entzündliche Geschwulst am Kopf, } \\
\text { starkes Herzklopfen }\end{array}$ \\
\hline & Büzürk & $\begin{array}{l}\text { Bauch, Gedärme, Zwerchfell, } \\
\text { unterer Rücken }\end{array}$ & $\begin{array}{l}\text { Kneifen im Unterleib, Koliken, } \\
\text { innere Unruhe }\end{array}$ \\
\hline & Neva & Knie, Lenden, Oberschenkel & Ischias, Lendenschmerz \\
\hline \multirow[t]{2}{*}{ Cholerische Entgleisung } & Isfahan & Kopf, Schulter, Arme, Hände & regt den Verstand an, schärft die Gedanken \\
\hline & Uşşak & Füsse & Schlaflosigkeit \\
\hline \multirow[t]{3}{*}{ Phlegmatische Entgleisung } & Rast & Kopf, Gesicht, Augen, Ohren & $\begin{array}{l}\text { Schlaganfälle mit „kalter", halbseitiger } \\
\text { Lähmung }\end{array}$ \\
\hline & Hicaz & Geschlechtsteile, Blase, Hüfte & Hüftschmerz, Dysurie, Impotenz, Frigidität \\
\hline & Buselik & Lenden, Oberschenkel, Hüften, After & Hüft- und Lendenschmerz, kalte Koliken \\
\hline \multirow[t]{3}{*}{ Melancholische Entgleisung } & Zirekfend & $\begin{array}{l}\text { Kopf, Brust, Lunge, Herz, Magen, Milz } \\
\text { Rücken-, Gelenkschmerzen, Koliken }\end{array}$ & Kopfschmerzen, Hemiparese, \\
\hline & Zengule & $\begin{array}{l}\text { Bauch unterhalb des Nabels, } \\
\text { Kreuz, Lenden, Nieren }\end{array}$ & $\begin{array}{l}\text { Liebesleiden, Delirium, } \\
\text { Herzleiden }\end{array}$ \\
\hline & Hüseyni & Unterschenkel & $\begin{array}{l}\text { 3-Tage-Wechselfieber, Magenbrennen, } \\
\text { Kältekrankheiten }\end{array}$ \\
\hline
\end{tabular}

sche Beschwerden nachhaltig zu lindern und dabei auf erstaunliche Weise kulturelle Schranken und Hörgewohnheiten zu überwinden.

\section{Literatur}

1. Bachmaier-Ekşi M: Konstitutionelle Altorientalische Musiktherapie. Ärztezeitschrift für Naturheilverfahren 2004;45(10):644-651.

2. Güvenç R O: Türklerde ve Dünyada Müsikle Ruhi Tedavinin Tarihcesi ve Günümüzdeki Durumu. - Doktora Tezi / T.C. Istanbul Universitesi Cerrahpasa T1p Fakültesi Psikiyatri Anabilim Dali 1985 (Geschichte und aktueller Stand der Therapie der Psyche durch Musik im türkischen und internationalen Kontext. Studientexte der Schule für Altorientalische Musik- und Kunsttherapie. Band 1. Eigenverlag, A-3924 Schloss Rosenau 1, 1989).

3. Tucek G: Orientalische Musik- und Kunsttherapie. Studientexte der Schule für Altorientalische Musik- und Kunsttherapie. Band 7. Eigenverlag, A-3924 Schloss Rosenau 1, ohne Jahresangabe.
4. Neubauer E: Arabische Anleitungen zur Musiktherapie. Institut für Geschichte der Arabisch-Islamischen Wissenschaften, Frankfurt a. M., 1990.

5. Chishti G.M.: The Traditional Healer's Handbook - A Classic Guide to the Medicine of Avicenna, Healing Arts Press, Vermont 1991.

6. Avicenna (Abu Ali al-Husayn ibn Abd Allah ibn Sina): The Canon of Medicine (al-Qanun fi'ltibb) (Übersetzung: O. Cameron Gruner und Mazar H. Shah). Kazi, Chicago, 1999.

7. Chishti SS, Chishti GM, Grotte BL, Abdullah HS, Igram C, Abdullah T, Ammat-Ul-Habib I, Thomson B: Unani Herbal Healing - Alternative Health Education, Web site des "American Institute of Unani Medicine",

www.unani.com, 2003.

8. Garvelmann F: Pflanzenheilkunde in der Humoralpathologie. Pflaum-Verlag, München 2000.

9. Porkert M: Neues Lehrbuch der chinesischen Diagnostik. Phainon-Verlag, Dinkelscherben 1993.

10. Garten, H. (1994): Akupunktur bei Inneren Erkrankungen. Hippokrates-Verlag, Stuttgart 1994.
11. Ahmad J, Qadeer A: Unani - The Science of Graeco-Arabic Medicine. Lustre Press, New Dehli 1998.

12. Pischinger A: Das System der Grundregulation. Haug-Verlag, Heidelberg 1990.

13. Brüggenwerth G, Gutjahr L, Kulka Th, Wilcken C, Machleidt, Hinrichs: Die Wirkung der Musik auf das Gehirn, in: Güvenc, R.O.: Türklerde ve Dünyada Müsikle Ruhi Tedavinin Tarihcesi ve Günümüzdeki Durumu - Doktora Tezi / T.C. Istanbul Universitesi Cerrahpasa T1p Fakültesi Psikiyatri Anabilim Dali 1985 (Studientexte der Schule für Altorientalische Musik- und Kunsttherapie. Band 1.Eigenverlag, A-3924 Schloss Rosenau 1, 1989).

\section{Korrespondenzadresse:}

Dr. med. Michael Bachmaier-Ekşi Praxis für Orientalische Medizin Mittenwalder Straße 20, DE-10961 Berlin bachmaier_eksi@yahoo.de 